\title{
Room-temperature continuous-wave upconverting micro- and nanolasing for bio-optofluidics
}

\author{
Angel Fernandez-Bravo ${ }^{*}{ }^{1}$, Liliana Moscardi ${ }^{1}$, Aaron M. Ross ${ }^{1}$, Guglielmo Lanzani ${ }^{1}$,Emory M. Chan ${ }^{2}$, Teri W. Odom ${ }^{3}$, P. \\ James Schuck ${ }^{4}$, and Francesco Scotognella ${ }^{1}$ \\ ${ }^{1}$ Dipartimento di Fisica - Politecnico di Milano P.zza Leonardo da Vinci 32, 20133 Milano \\ ${ }^{2}$ The molecular Foundry, Lawrence Berkeley National Laboratory, 94720, Berkeley, California \\ ${ }^{3}$ Department of Chemistry, Northwestern University, Evanston, IL, USA. 5 \\ ${ }^{4}$ Department of Mechanical Engineering, Columbia University, New York, NY, USA
}

\begin{abstract}
Nanolasers that operate under the continuous-wave pump and are robust in diverse environments will make possible compact optoelectronic devices, biomedical imaging, and large-scale quantum photonics. However, current nanolasers require low temperatures or pulsed excitation because their small mode volumes severely limit gain relative to cavity loss. Here, I will present continuous-wave upconverting micro- and nanolasing at room temperature with record-low thresholds and high photostability. I will explore the future implications of such a low-threshold laser for optofluidics.
\end{abstract}

Nanolasers that operate under continuous-wave pump and are robust in diverse environments will make possible compact optoelectronic devices, biomedical imaging, and large-scale quantum photonics. However, current nanolasers require low temperatures or pulsed excitation because their small mode volumes severely limit gain relative to cavity loss. Here, I will present continuouswave upconverting micro- and nanolasing at room temperature with record-low thresholds and high photostability. I will talk about the coupling between $\mathrm{Tm}^{3+}$-based energy-looping nanoparticles (ELNPs) and stand-alone optical microcavities with implications in deep-tissue imaging ${ }^{1,2}$.

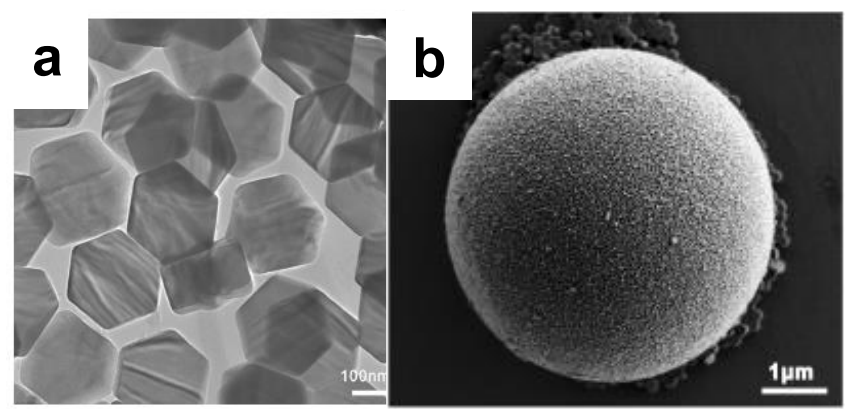

Figure 1 - a. TEM image of upconversion nanoparticles used to coat microresonators. b. SEM image of a microsphere.

I will also cover how to achieve directional lasing to enhance interactions at the nanoscale and selective singlemode lasing from $\mathrm{Yb}^{3+} / \mathrm{Er}^{3+}$-co-doped upconverting nanoparticles conformally coated on silver nanopillar arrays supporting sharp lattice plasmon modes, Figure 2.

The intense electromagnetic nearfields resulted in a threshold of $29 \mathrm{~W} / \mathrm{cm} 2$, orders of magnitude lower than other small lasers ${ }^{3}$. Micron-sized lasers fabricated from upconverting nanoparticles (UCNP) coupled to whispering gallery mode (WGM) microresonators can exhibit continuous-wave anti-Stokes lasing useful for tracking cells, environmental sensing, and coherent stimulation of biological activity. The integration of these microlasers into organisms and microelectronics requires even smaller diameters, however, which raises threshold pump powers beyond practical limits for biological applications. To meet the need for low lasing thresholds and high-fidelity fabrication methods, we use correlative optical and electron microscopy, Figure 2, to uncover the nanoparticle assembly process and structural factors that determine efficient upconverted lasing.

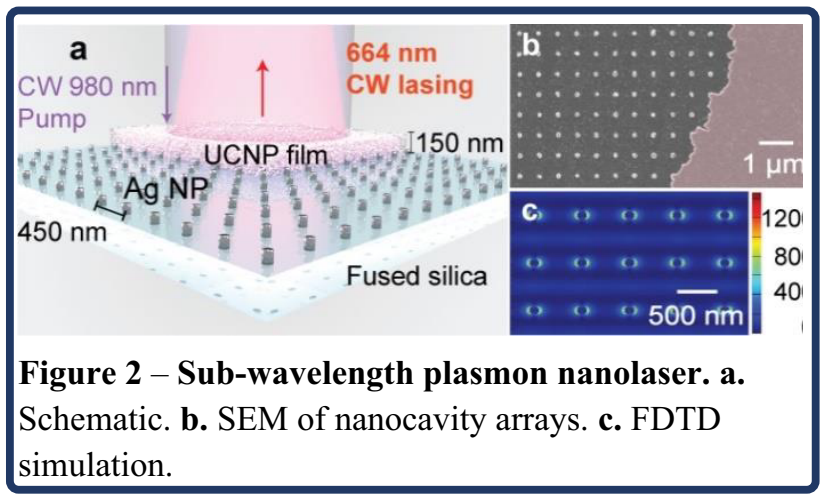

* Corresponding author: angel.fernandez@polimi.it 


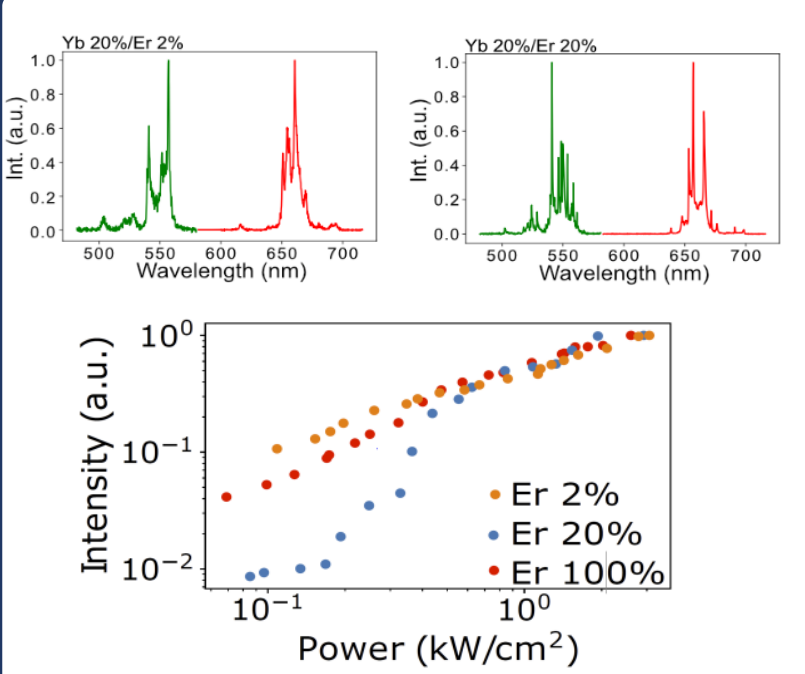

Figure 3 - Upper panels: Spectra with changing concentrations of $\mathrm{Er}^{3+}$ ions in the crystal lattices use for lasing at low thresholds. Lower panel: Light-Light curves for 3 lanthanide compositions with changing spontaneous emission coupling (beta) factors, showing thresholdless lasing behavior.

I will explore the future implications of such a lowthreshold laser and the potential paradigm change they can bring to, deep-tissue interrogation, microfluidic design and bio-optofluidics. Here we show upconverting microlasing with high photostability under continuous wave (CW) pump enabled by photonic microcavities. Solution-processed UCNPs at a submonolayer thickness can be coated on biocompatible polymeric (polystyrene) cavities. After pumping with a $\mathrm{CW}$ laser at NIR wavelength, we observed lasing in the visible regime ca. 550 and $650 \mathrm{~nm}$ at room temperature. We observe lasing at thresholds in the order of 100's of W/ $\mathrm{cm}^{2}$ which helps preserve biological tissues and species, Figure 3. This, along with the exceptionally large spontaneous emission coupling and low lasing threshold will be critical in the development of future biologically compatible optomechanical traps, and bio-integrated sensors.

We demonstrate that the WGM signatures of these upconverting microlasers can be imaged and distinguished through tissue-mimicking phantoms ${ }^{2,4}$. These advances will enable the fabrication of more efficient upconverting lasers for imaging, sensing, and actuation in optically complex environments.

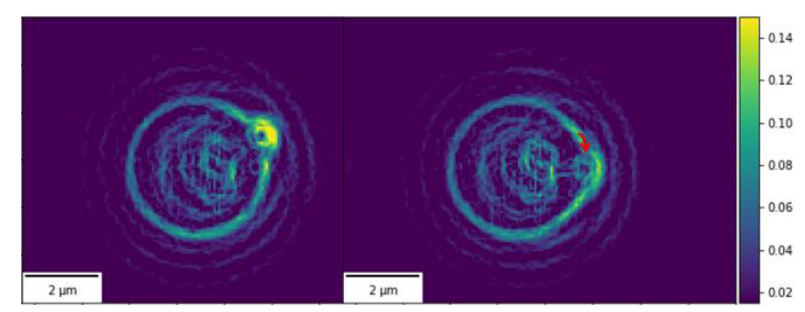

Figure 4 - Segmented images show proteins orbiting around a stand-alone micrometer size optofluidic trap.

Additionally, the solid-state laser showed high photostability that allows for long experiments with biological samples with no observed degradation. I will extend the $\mathrm{Yb}^{3+} / \mathrm{Er}^{3+}$-co-doped lasers to stand alone microcavities to fabricate optomechanical traps for biologically relevant species, shown in Figure 4. The result of these microscopic optical traps is a fine control of nanometer scale species in a reduced volume, fully controlled by light.

\section{References}

1. Levy, E. S. et al. Energy-Looping Nanoparticles: Harnessing Excited-State Absorption for Deep-Tissue Imaging. ACS Nano 10, (2016).

2. Fernandez-Bravo, A. et al. Continuous-wave upconverting nanoparticle microlasers. Nat. Nanotechnol. 13, (2018).

3. Fernandez-Bravo, A. et al. Ultralow-threshold, continuous-wave upconverting lasing from subwavelength plasmons. Nat. Mater. 18, 1172-1176 (2019).

4. Liu, Y. et al. Controlled Assembly of Upconverting Nanoparticles for Low-Threshold Microlasers and Their Imaging in Scattering Media. ACS Nano 14, 1508-1519 (2020). 